\title{
Accelerating the Adoption of Virtual Traffic Lights through Policy Decisions
}

\author{
Wantanee Viriyasitavat \\ Faculty of Information and \\ Communication Technology \\ Mahidol University, Bangkok, Thailand \\ wantanee.vir@mahidol.ac.th
}

\author{
Juan M. Roldan \\ Engineering and Public Policy \\ Carnegie Mellon University \\ Pittsburgh, PA 15213-3890 \\ jroldan@andrew.cmu.edu
}

\author{
Ozan K. Tonguz \\ Electrical and Computer Engineering \\ Carnegie Mellon University \\ Pittsburgh, PA 15213-3890 \\ tonguz@ece.cmu.edu
}

\begin{abstract}
A new technology known as Virtual Traffic Lights (VTL) was recently proposed as a self-organizing new paradigm for traffic management. This new technology uses the vehicle-tovehicle (V2V) communications as its premise. VTL can revolutionize traffic management in urban areas as it can substantially reduce commute time of urban workers, increase productivity, and lead to a greener environment. In a VTL environment, vehicles self-organize to elect a leader which serves as a virtual traffic light to decide the right of way at that intersection, thus replacing the current physical traffic lights. Implementing VTL technology with partial penetration, however, is an outstanding issue that needs to be addressed. This paper addresses this issue by proposing a co-existence model whereby VTL equipped vehicles can co-exist with vehicles that do not have VTL. Simulation results show that the transition model proposed here could provide drivers with strong incentives to adopt the VTL technology.
\end{abstract}

Keywords-vehicle-to-vehicle communications; intelligent transportation systems; virtual traffic lights

\section{INTRODUCTION}

Vehicle to vehicle communications (V2V) using Dedicated Short-Range Communication (DSRC) technology in the 5.9 $\mathrm{GHz}$ band have facilitated the development of Virtual Traffic Lights (VTLs) [1]. In a VTL environment, vehicles selforganize to manage the right of way at intersections, obviating the use of current traffic lights, optimizing travel times of urban workers, increasing productivity, reducing carbon foot print of vehicles, and leading to a greener environment. However, given that the introduction of DSRC and VTL technology might happen gradually (with initial penetration rates in the vicinity of $5-10 \%$ ), policies should be designed to pave the way for a smooth transition from the current traffic light system to a VTL system [2]. In this paper, we propose such a coexistence model for the transition period whereby VTLequipped vehicles can benefit from the VTL technology while they co-exist with vehicles that do not have VTL equipment. The co-existence model will be used to show how certain policy decisions during designated periods (e.g., during rush hours) can expedite and provide incentives for adopting the VTL technology by rewarding VTL vehicles while slightly penalizing the non-VTL vehicles.

This research was supported in part by the T-SET University Transportation Center sponsored by the US Department of Transportation under Grant No. DTRT12-G-UTC11.

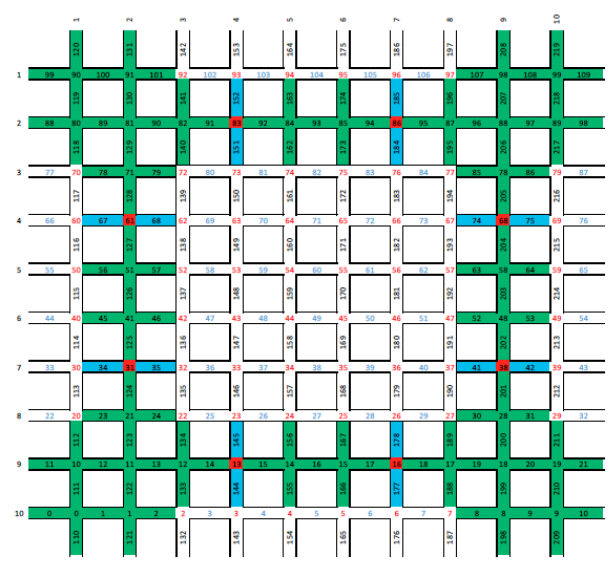

Fig 1: Example of a road network in which some streets (highlighted in green color) and intersections (also in green color) are reserved for the exclusive use of VTL-equipped vehicles during rush hours.

\section{VTL COEXISTENCE MODEL}

The coexistence model proposed in this paper is inspired by the High Occupancy Vehicle (HOV) lane paradigm whereby some lanes on a highway, for instance, are reserved for the exclusive use of vehicles carrying passengers. An example of the proposed VTL coexistence model is shown in Figure 1. Observe from the figure that streets and intersections that are high- lighted in green color are reserved exclusively for the VTL- equipped vehicles. The intersections on the VTLexclusive streets, with an exception of the intersections highlighted in red color, also operate based on the VTL paradigm [1]. In other words, the current traffic lights at those intersections will be replaced by the virtual traffic lights; vehicles that approach one of these intersections will communicate among themselves and decide to elect a leader that will manage the traffic flow at that intersection. Note that the physical traffic lights are still being used at other intersections including the ones highlighted in red color. The intersections highlighted in red color therefore manage both VTL-equipped vehicles and vehicles that do not have VTL equipment (i.e., non-VTL vehicles). These intersections are necessary as they allow non- VTL vehicles to cross the VTL exclusive streets.

By reserving some streets exclusively for the use of VTL vehicles, it is clear that the VTL-equipped vehicles will benefit from this policy enforcement while the non-VTL vehicles get 
penalized, as they are restricted to use only some of the roads. Benefits in terms of reduction in travel time of VTL vehicles can be attributed to two reasons: i) the VTL vehicles may travel at a higher speed on the VTL-exclusive streets since these streets are of a restricted use and tend to have less traffic and ii) a more effective traffic management at intersections provided by VTL reduces the waiting time of VTL-equipped vehicles to cross the intersections and thus reduce the overall travel time. In turn, this setting may penalize and increase the travel time of the non-VTL vehicles; i.e., the non-VTL vehicles might have to travel over a longer distance to avoid the exclusive streets and experience higher level of traffic congestion even on the normal roads.

\section{PRELIMINARY STUDY}

We resort to SUMO traffic simulator [3] to study the tradeoff mentioned in the previous section. A 10x10 Manhattan Grid network with two-way one-lane streets is assumed in the simulations and three configurations with different number of VTL-exclusive streets are considered as shown in Figure 2. In all configurations, an evening rush hour traffic pattern is assumed whereby all vehicles originate from a point within the $3 \times 3$ inner square grid (indicated by the dotted red rectangles in the figures) and they move toward their destinations located on the boundary of the grid network. In the simulations, one vehicle is generated each second for a period of 1,500 seconds. With this generation rate, the number of vehicles in the road network stays almost constant at 250 during the peak period. While the network topology assumed here might not resemble the actual road topology in real cities, it serves as a proof-ofconcept example in our preliminary study to determine the feasibility of the proposed VTL coexistence model.

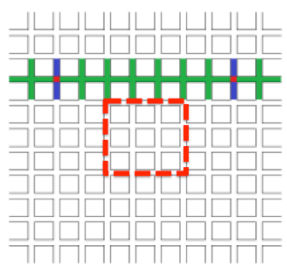

Configuration \#1

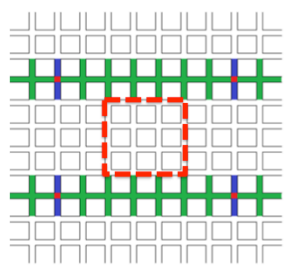

Configuration \#2

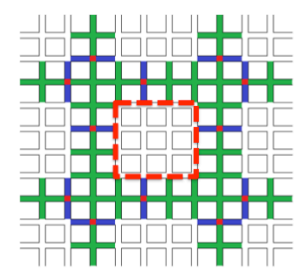

Configuration \#3
Fig 2: Road Configurations considered in the study.

Figure 3 shows the simulation results in terms of mean travel speed of VTL and non-VTL vehicles. The red horizontal line indicates the average speed of 13:2 $\mathrm{mph}$ when the current physical traffic light system is implemented and no streets are reserved for VTL exclusive use. Observe that when the drivers start to adopt the VTL technology, the scheme already provides significant benefits to VTL-equipped vehicle and penalizes the non-VTL vehicles even in the scenario with a single VTLexclusive street (i.e., the average travel speeds of VTLequipped and non-VTL vehicles changes by $+14 \%$ and $-39 \%$, respectively). This kind of reward and penalty system provides strong incentives for adopting the VTL technology.

Nevertheless, as more consumers adopt the VTL technology, the advantage provided by VTL-exclusive roads diminishes (i.e., VTL-equipped vehicles do not gain any reduction in travel time in a scenario where a large fraction of
VTLvehicles and only a few VTL-exclusive streets). This is because the large volume of VTL-equipped vehicles causes traffic congestion on the VTL-exclusive streets and traveling on these streets is no longer beneficial to the VTL-vehicles.

In such scenarios, it might be desirable to reserve additional streets for VTL exclusive use (e.g., configurations 2 and 3 in Figure 2). While the results shown here indicate how different road configurations affect the overall travel speed experienced by vehicles, a more detailed investigation on this topic is necessary before the policy makers come up with an appropriate coexistence model that can be used during the transition period.

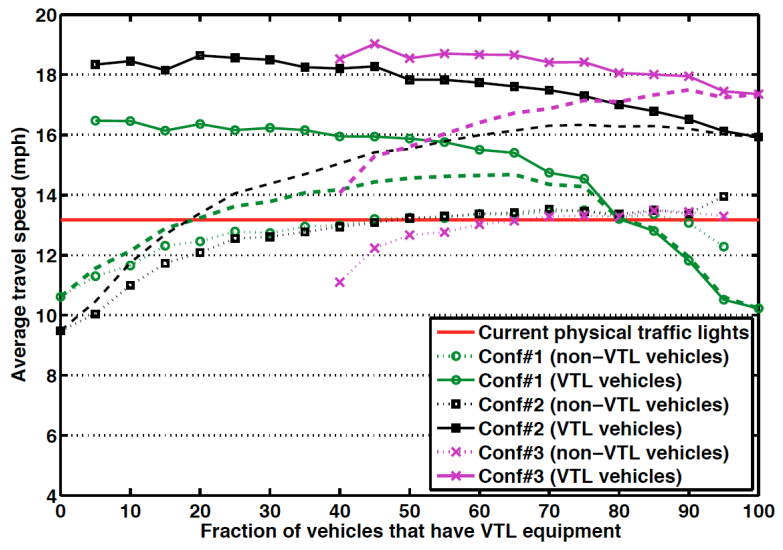

Fig 3: Average travel time of VTL-equipped and non-VTL-equipped vehicles when three different coexistence models shown in Figure 2 are used. The dotted lines represent the weighted average when both VTL-equipped and non-VTL vehicles are considered.

\section{CONCLUSION}

Vehicle-to-vehicle (V2V) communications have facilitated the development of self-organizing traffic control, namely Virtual Traffic Lights (VTL). However, one of the major concerns in implementing the VTL system is the fact that all vehicles are required to be equipped with radio devices for $\mathrm{V} 2 \mathrm{~V}$ communications and VTL equipment. This paper addresses this outstanding issue by proposing a transition model in which VTL-vehicles benefit from the VTL technology while coexisting and sharing the streets with current non-VTL vehicles. The proposed co-existence model seems very promising as it shows how judicious policy decisions by Department of Transportations of different countries could expedite the adoption of the Virtual Traffic Lights technology. Simulation results have shown that the proposed model could provide the drivers of vehicles strong incentives to adopt the VTL technology much faster than otherwise.

\section{REFERENCES}

[1] M. Ferreira, R. Fernandes, H. Conceicao, W. Viriyasitavat, and O. K. Tonguz, "Self-organized traffic control," in the ACM international workshop on VehiculAr InterNETworking (VANET), 2010, pp. 85-90.

[2] Y. Toor, P. Muhlethaler, and A. Laouiti, "Vehicle ad hoc networks: applications and related technical issues," IEEE Communications Surveys Tutorials, vol. 10, no. 3, pp. 74-88, 2008.

[3] M. Behrisch, L. Bieker, J. Erdmann, and D. Krajzewicz, "Sumo-simulation of urban mobility: An overview," in SIMUL 2011, The Third International Conference on Advances in System Simulation, Barcelona, Spain, October 2011, pp. 63-68. 